$\underline{\text { Assiut Vet. Med. J. Vol. } 59 \text { No. } 136 \text { January } 2013}$

\title{
DIAGNOSTIC STUDY OF PARASITIC WORMS AND INTESTINAL PROTOZOA IN CATTLE
}

M.H. HASAN and W.K. KHALAF

Department of Microbiology , College of Veterinary Medicine, University of Mosul , Mosul-Iraq

\section{ABSTRACT}

Received at: 18/10/2012

Accepted: / /
This study included diagnosis of some parasitic worms and intestinal protozoa which infected the cattle, through the examination of (325) fecal samples which where collected randomly from the different regions in Mosul city, from the animals in different ages, from October (2011) to March (2012). The total percentage of infection with different species of parasites was $(53.53 \%)$. The percentages have been influenced through the months of the study. The high percentage of infection appeared in January was (76.66\%) and low percentage appeared in February was $(23.68 \%)$. The high percentage of infection in intestinal protozoa was $(60.91 \%)$, while the percentage of infection in Nematoda was $(49.42 \%)$ then the percentage in Trematoda and Cestoda were (17.24\%), (1.72\%) respectively. In this study has been diagnosed (12) Genera in Nematoda. The infection of Capillaria bovis was the first record in Mosul city, also in this study has been described the adult worm of Toxocara vitulorum which found in the feces of infected calves, that is not mentioned previously in Mosul city. Also has been diagnosed (5) species in Trematoda, the high percentage of infection is Dicrocoelium dendriticum was $(8.04 \%)$, while by Cestoda the percentage was $(1.72 \%)$ in one species Moniezia expansa. The results indicated the (5) species by intestinal protozoa which are infected the cattle, the high percentage of infection in Cryptosporidium spp. was (35.05\%). The severity of infection with Nematoda was high percentage of inflection in low grade, then severe and moderate grade.

Key words: Parasitic worms, Intestinal protozoa, Cattle, Diagnosis.

دراسة تشخيصية للايدان الطفيلية والأوليات المعوية في الأبقار

منال حمادي حسن ، وعد خالد خلف

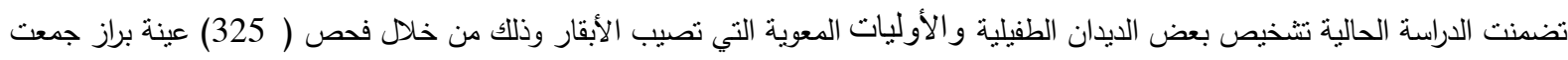
عشوائيا من مناطق مختلفة من مدينة الموصل وبأعمار مختلفت خلال المدة من شهر تشرين الأول (2011) ولغاية شهر آذار (2012). حيث ظهرت النسبة الكلية للإصابة بمختلف أنواع الطفيليات ( 53.53 \%) وتباينت نسب الإصابة حسب أنثهر الدراسة، فقد سجلت أعلى نسبة إصابة في شهر كانون الثاني إذ بلغت ( 76.66 \% وأدنى نسبة إصابة سجلت في شهر شباط بلغت ( 23.68 \% \%). وقد سجلت أعلى نسبة للإصابة بالاوالي المعوية إذ بلغت ( 60.91 \%) ثم الإصابة بالديدان الأسطوانية إذ بلغت ( 49.42 \% \% 4 \% وتلتها الإصابة بديدان المثقوبات والثريطيات إذ بلغت ( 17.24 \%)، (1.72 \%) على التوالي. وتم تشخيص ( 12) جنس من الديدان الأسطوانية حيث كان تسجيل الإصابة

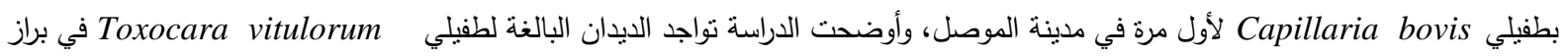
العجول المصابة والتي لم يثار إليها من قبل في مدينة الموصل. وتم نشخيص خمسة أنواع من ديدان المثقوبات حيث كان أعلى نسبة إصابة هو

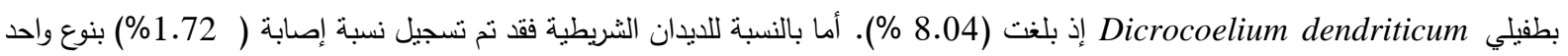

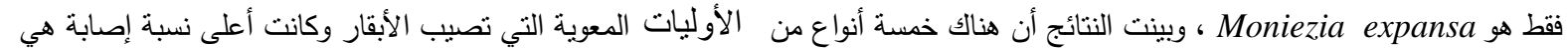
بطفيلي .Cryptosporidium spp إذ بلغت (35.05 \%). وعند تقدير شدة الإصابة بالديدان الأسطوانية أوضحت النتائج أن أعلى نسبة إصابة كانت من النوع الخفيف ثم الإصابة الثنديدة والمتوسطة. 
Assiut Vet. Med. J. Vol. 59 No. 136 January 2013

\section{INTRODUCTION}

\section{المقدمة}

تصاب العديد من الحيوانات الحقلية ومن ضمنها الأبقار بأنواع مختلفة من طفيليات المعدة والأمعاء، وتعد الإصابة بهذه الطفيليات من الأمراض الواسعة الانتثار في العالم ( Vercruysse and Claerebout, 2001; Regassa et al., 2006 )، وتكمن أهمية هذه الطفيليات في الحيوانات الحقلية لما تسبيه من خسائر اقتصادية ناتجة عن اعتلال صحة الحيوان وتأخير في نمو الحيوانات المصابة وانخفاض مستوى الإنتاج هذا من جهة ، ومن جهة أخرى ارتفاع تكاليف المعالجة من إحدى المشاكل الهامة التي نواجه القائمين على تتمية الثروة الحيوانية ورعايتها .(Iqbal, et al., 1993; Lebbie, et al., 1994; Perry and Randolpph, 1999; Ather, et al., 2011)

اهتم العديد من الباحثين في مختلف أنحاء العالم بوبائية الإصابات الطفيلية في القناة الهضمية للأبقار التي تثشل الديدان الاسطوانية والثريطية

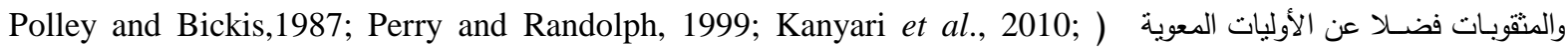
.(Safavi et al., 2011

في العراق فقد نم تثخيص العديد من أنواع ديدان المعدة والأمعاء في الأبقار في مناطق مختلفة من القطر، وكانت أول دراسة في هذا الصدد هي

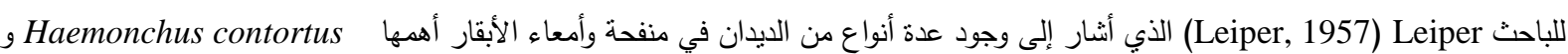
Ostertagia ostertagi المعدة 43.6 \% وفي الأمعاء 56.4 \% ونم تسجيل 14 نوعا من ديدان المعدة والأمعاء وكان أكثر الأنواع المسجلة انتشارا هي (Al-Dulimi et al., 1986) Haemonchus contortus نسبة خمج في الأبقار بلغت 42.8 \% لطفيل الدودة الكبدية Al-Baz et al., 2002) Fasciola hepatica). وفي دراسة أجريت في محافظة

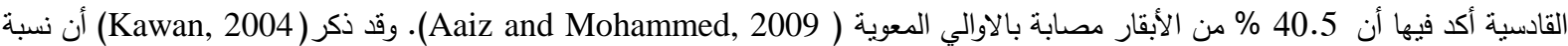

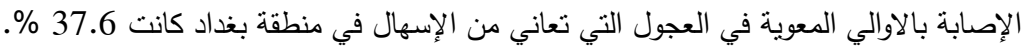

في مدينة الموصل ، سجلت (Al-Farwachi, 2000) في دراستها عن الديدان الداخلية في الأبقار في محافظة نينوى أعلى نسبة إصابة بالديدان

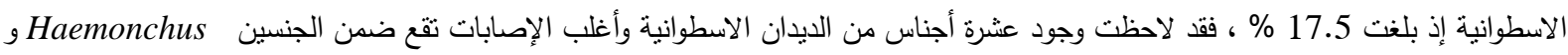
Ostertagia 33.9 \% في الأبقار المذبوحة (إبراهيم، 2005) ، بينما ذكر (Abdulhameed, et al., 2012) أن النسبة الكلية لإصابة العجول بالعديد من الطفيليات الاسطوانية والثريطية بلغت 60.99 \% في منطقة كوكجلي. ونسبة إصابة العجول بالاوالي المعوية في محافظة نينوى سجلت نسبة 54.5 \% من قبل الباحثين (Hasan, et al., 2010).

ولتعزيز الدراسات على الأبقار المصابة بطفيليات المعدة والأمعاء ، أجريت هذه الدراسة التي هدفها تثخيص الأنواع المختلفة من طفيليات المعدة

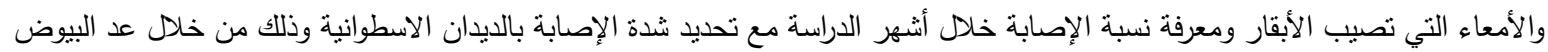
لكل 1 غم من البراز.

\section{MATERIALS and METHODS} المواد وطرائق العمل

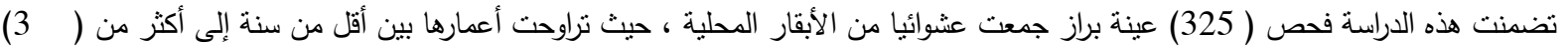

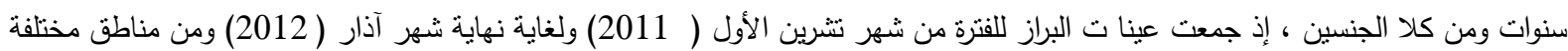
من مدينة الموصل تضمنت ( الرحمانية ، الرشيدية ، الآربجية ، حقول كلية الزراعة ، حي التحرير ، بارمجة ، بعويزة ، كوكجلي ، الجماسة ، الإل حي

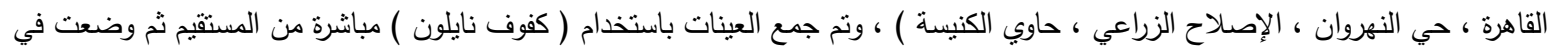

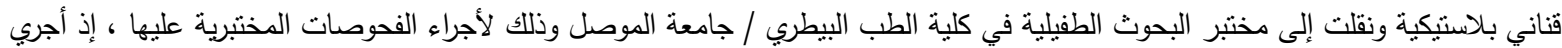

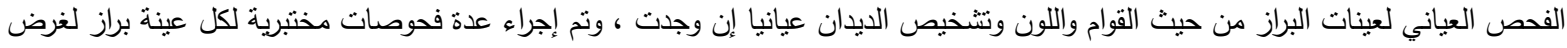
التحري عن بيوض الديدان الطفيلية والأوليات المعوية والتي شملت: مسحات مباشرة من البراز ( Foreyt, 2001) ، مسحات مبات مباثرة مع إضافة 


\section{$\underline{\text { Assiut Vet. Med. J. Vol. } 59 \text { No. } 136 \text { January } 2013}$}

صبغة الايودين (Baron et al., 1984) ، طريقة التطويف بالمحلول السكري ( Urquhart et al., 2003 ( بالصبغة الصامدة للحامض المحورة (Baron et al., 1994) ، طريقة الترسيب (Kassai, 1999) ، وتم حساب عدد البيوض للايدان الاسطوانية

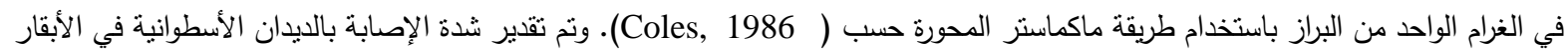

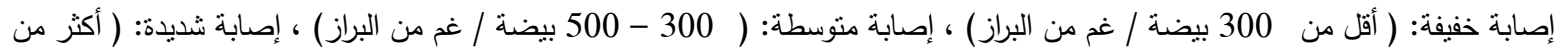

500 بيضة / غم من البراز)، ( Soulsby, 1986; Shah-Fischer and Say, 1989 ) وتم تشخيص الديدان البالغة لطفيلي vitulorum بالاعتماد على المواصفات التي ذكرها ( Soulsby, 1986; Urquhart et al., 2003 ) ، وتم تصوير بيوض الطفيليات باستخدام الكاميرا الرقمية. عند تحليل النتائج إحصائيا باستخدام برنامج Sigma Stat 3.0 تم استخدام اختبار مربع كاي وعند مستوى معنوية (p<0.05).

\section{RESULTS}

النتائسج

أظهرت نتائج الدراسة أن نسبة الإصابة الكلية بالايدان الطفيلية والأوليات المعوية في الأبقار في المناطق الددروسة كانت ( 53.53 \% وسجلت أعلى نسبة إصابة في شهر كانون الثاني وبنسبة ( 76.66 \%) ، في حين كانت أقل نسبة إصابة في شهر شباط وبنسبة ( 23.68 \%) ، جدول رقم (1).

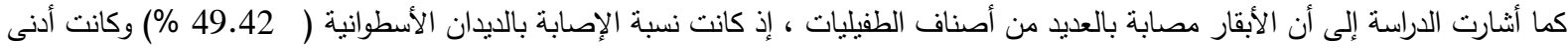

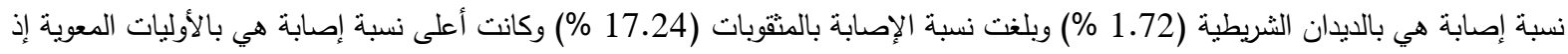

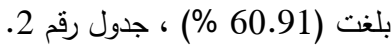

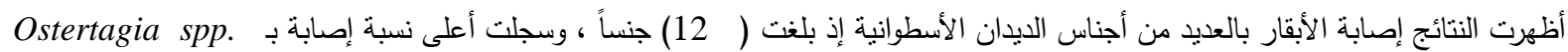

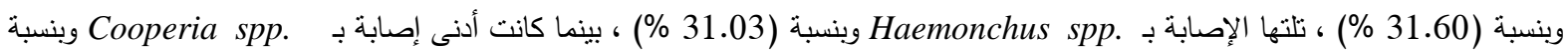
(0.57 (0.57) ، جدول رقم (3) ، شكل (1).

أثنارت الدراسة إلى وجود الديدان البالغة نوع Toxocara vitulorum في براز الأبقار التي وجدت أثناء فحص براز الأبقار عيانيا ، حيث بلغت

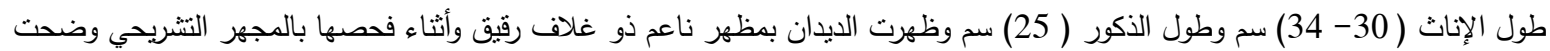
المقدمة الأمامية للايدان بامتلاكها على ثلاث شفاه واضحة تتسع عند قواعدها وتضيق عند نهايتها الأمامية. ( شكل 2، 3ن 3)

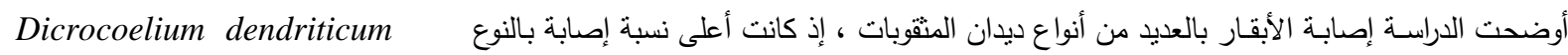

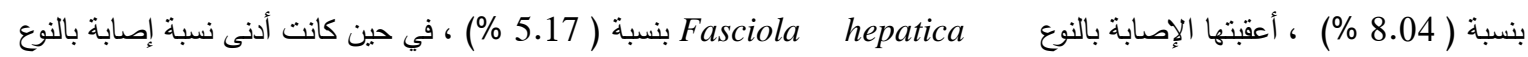
Moniezia expansa بنسبة (20histosoma bovis وبنسبة (1.72 \%)، (جدول 4 ، شكل 4).

Cryptosporidium spp. كما أثارت الدراسة إلى إصابة الأبقار بالعديد من أنواع الأوليات المعوية ، إذ سجلت أعلى نسبة إصابة بطفيلي

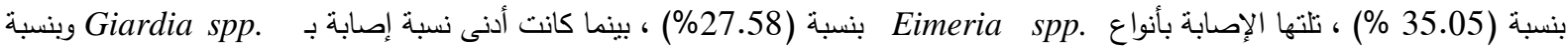
(4.59 \%) ، جدول (5) ، شكل (5).

عند تقدير شدة الإصابة بالديدان الأسطوانية وذلك من خلال حساب أعداد البيوض المطروحة بالغرام الواحد من البراز ، أظهرت النتائج أن نسبة

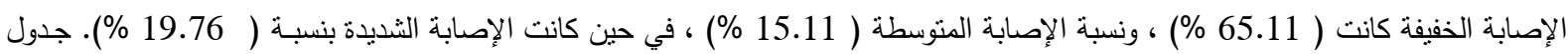


$\underline{\text { Assiut Vet. Med. J. Vol. } 59 \text { No. } 136 \text { January } 2013}$

جدول (1 ) : أعداد ونسب الإصابة بالديدان الطفيلية والأوليات المعوية في الأبقار وحسب أشهر الدراسة.

\begin{tabular}{|c|c|c|c|}
\hline نسبة الإصابة \% & عدد الحيوانات المصابة & عدد الحيوانات المفحوصة & الثهر \\
\hline a,b 61.29 & 38 & 62 & تشرين الأول 2011 \\
\hline a,b 58.69 & 27 & 46 & تشرين الثاني \\
\hline a 52.63 & 20 & 38 & كانون الأول \\
\hline b 76.66 & 46 & 60 & كانون الثاني 2012 \\
\hline c 23.68 & 18 & 76 & شباط \\
\hline $\begin{array}{ll}\mathbf{a , b} & 58.13 \\
\end{array}$ & 25 & 43 & آذار \\
\hline 53.53 & 174 & 325 & المجموع \\
\hline
\end{tabular}

جدول (2) : أعداد ونسب الإصابة بأصناف الديدان الطفيلية والأوليات المعوية في الأبقار .

\begin{tabular}{|c|c|c|}
\hline النسبة المئوية \% & عدد الحيوانات المصابة & أصناف الطفيليات \\
\hline a 49.42 & 86 & الاسطوانية \\
\hline b 1.72 & 3 & الشريطية \\
\hline${ }^{\mathrm{c}} 17.24$ & 30 & المثقوبات \\
\hline a 60.91 & 106 & الاوالي \\
\hline 53.53 & 174 & المجموع \\
\hline
\end{tabular}

الأحرف الدختلفة تثير إلى وجود فرق معنوي عند مستوى معنوية (P<0.05).

جدول (3) : أعداد ونسب الإصابة بأنواع الديدان الاسطوانية في الأبقار.

\begin{tabular}{rrr}
\hline ن نو الطفيلي \\
\hline 31.03 & 54 & Haemonchus spp. \\
\hline 31.60 & 55 & Ostertagia spp. \\
\hline 14.94 & 26 & Trichostrongylus spp. \\
\hline 11.49 & 20 & Strongyloides papillosus \\
\hline 10.34 & 18 & Chabertia ovina \\
\hline 1.14 & 2 & Capillaria bovis \\
\hline 1.14 & 2 & Trichuris spp. \\
\hline 10.34 & 18 & Oesophagostomum radiatum \\
\hline 6.89 & 12 & Nematodirus spp. \\
\hline 4.59 & 8 & Toxocara vitulorum \\
\hline 2.87 & 5 & Bunostomum spp. \\
\hline 0.57 & 1 & Cooperia spp. \\
\hline
\end{tabular}


$\underline{\text { Assiut Vet. Med. J. Vol. } 59 \text { No. } 136 \text { January } 2013}$

جدول (4) : أعداد ونسب الإصابة بأنواع ديدان المثقوبات والديدان الثريطية في الأبقار .

\begin{tabular}{ccr}
\hline نوع الطفيلي \\
\hline \% عدبة الحيوانات المصابة الإصابة & 9 & Fasciola hepatica \\
\hline 5.17 & 5 & Paramphistomum cervi \\
\hline 2.87 & 14 & Dicrocoelium dendriticum \\
\hline 8.04 & 3 & Eurytrema pancreaticum \\
\hline 1.72 & 1 & Schistosoma bovis \\
\hline 0.57 & 3 & Moniezia expansa \\
\hline 1.72 &
\end{tabular}

جدول (5) : أعداد ونسب الإصابة بأنواع الأوالي المعوية في الأبقار

\begin{tabular}{ccr}
\hline \% نوع الطفيلي \\
\hline \% عدد الحيوانات المصابة & 48 & Eimeria spp. \\
\hline 27.58 & 61 & Cryptosporidium spp. \\
\hline 35.05 & 8 & Giardia spp. \\
\hline 4.59 & 15 & Balantidium coli \\
\hline 8.62 & 30 & Buxtenella sulcata \\
\hline 17.24 & &
\end{tabular}

جدول (6) نسب وشدة الإصابة بأنواع الايدان الأسطوانية في الأبقار

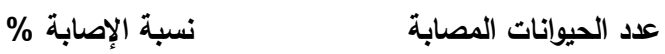

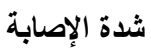

${ }^{\text {a }} 66.11$

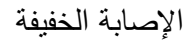

b 15.11

13

الإصابة المتوسطة

b 19.76

17

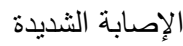

49.42

86

المجموع

الأحرف المختلفة تشير إلى وجود فرق معنوي عند مستوى معنوية (P<0.05)

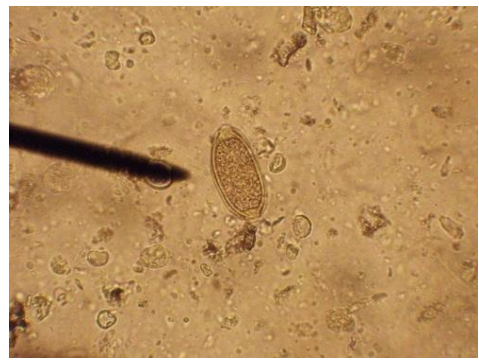

X40 Capillaria bovis

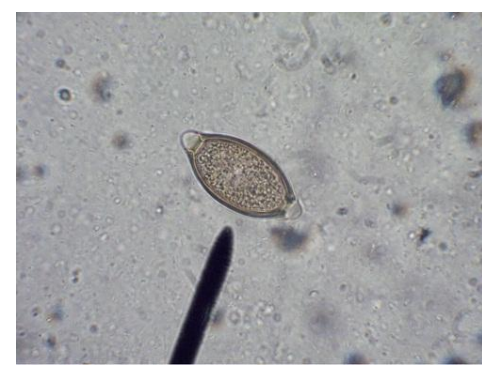

X40 Trichuris spp.bovis

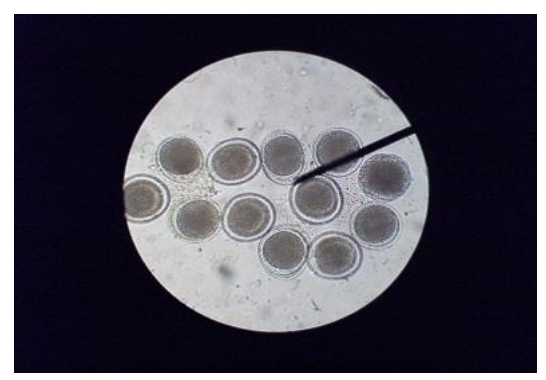

X10 Toxocara vitulorum

شكل (1) : بيوض بعض طفيليات الديدان الأسطوانية. 
$\underline{\text { Assiut Vet. Med. J. Vol. } 59 \text { No. } 136 \text { January } 2013}$

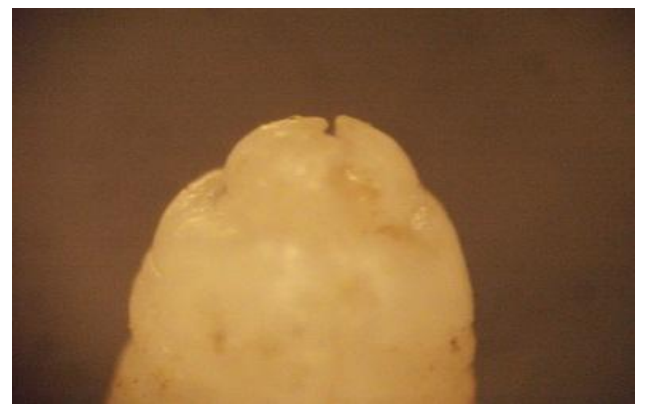

شكل(3): النهاية الأمامية لدودة Toxocara vitulorum أثناء

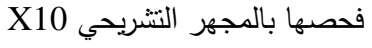

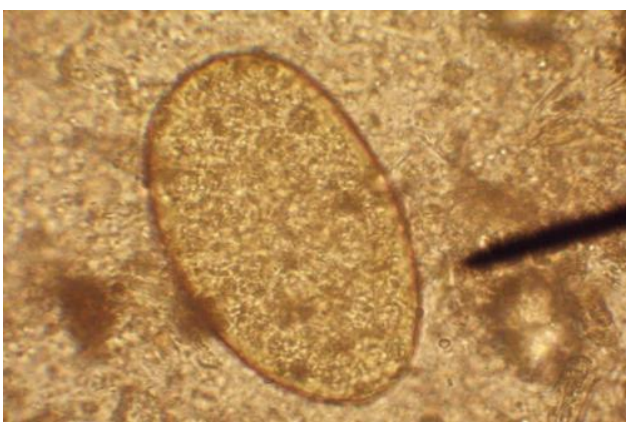

Fasciola hepatica

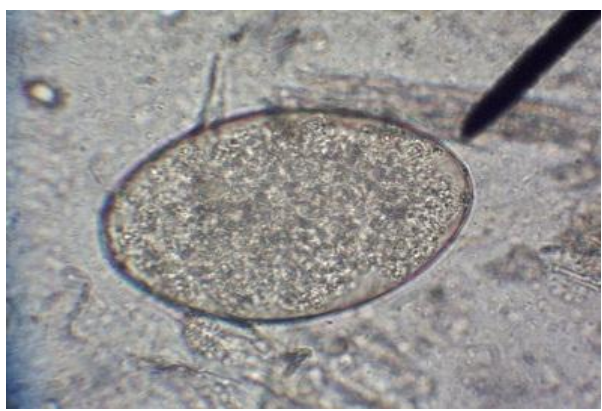

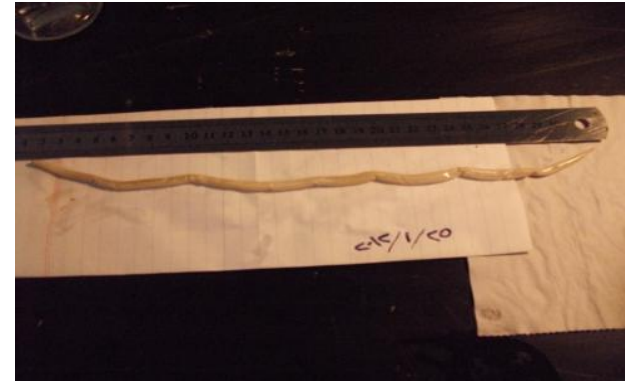

Toxocara vitulorum شكل(2) : الدودة البالغة لطفيلي

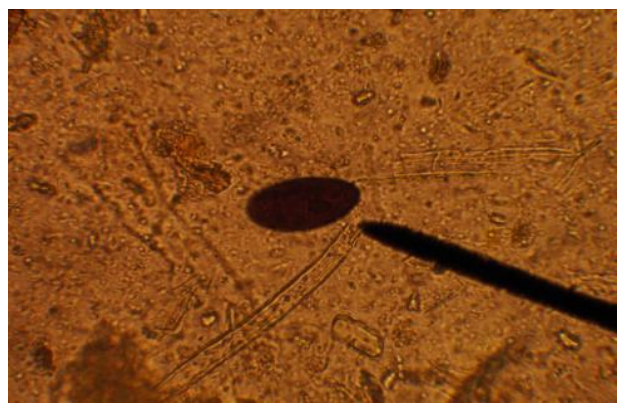

Dicrocoelium dendriticum

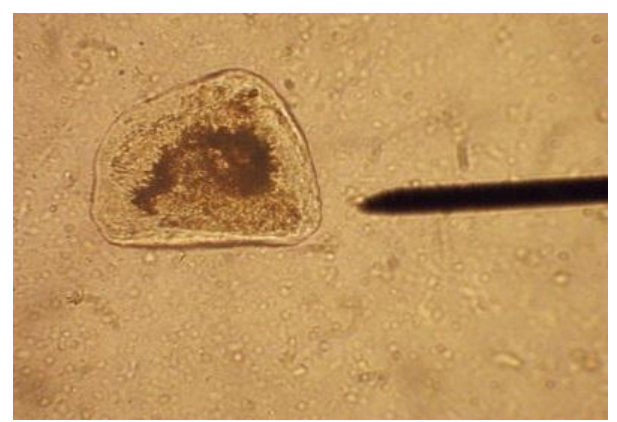

شكل(4) : بيوض بعض طفيليات ديدان المنقوبات وبيضة طفيلي Moniezia expansa. (X40)

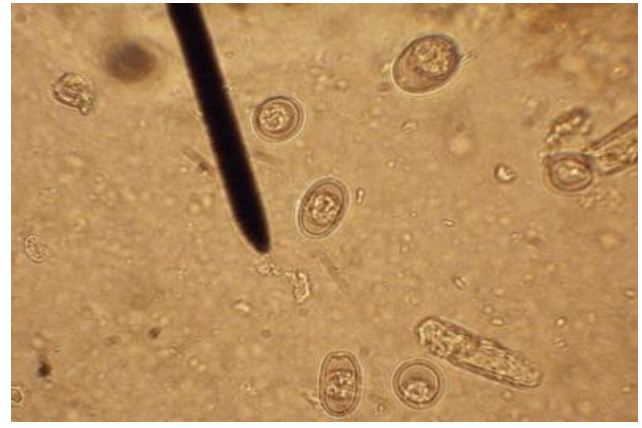

X40 Eimeria spp.

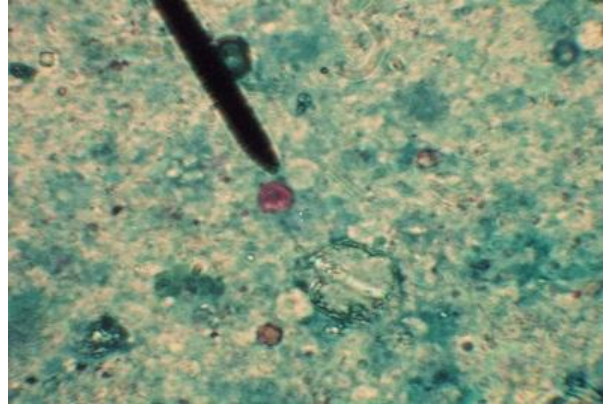

X100 Cryptosporidium spp.

المصبوغة بالصبغة الصامدة للحامض المحورة

$$
\text { شكل (5) أكياس بيض بعض الأوليات المعوية }
$$


$\underline{\text { Assiut Vet. Med. J. Vol. } 59 \text { No. } 136 \text { January } 2013}$

\section{DISCUSSION}

المناقثة

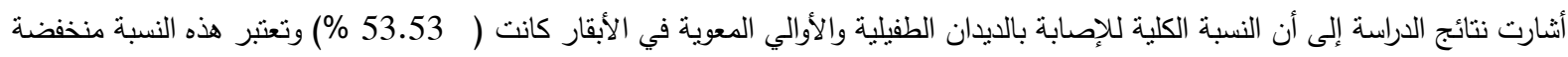

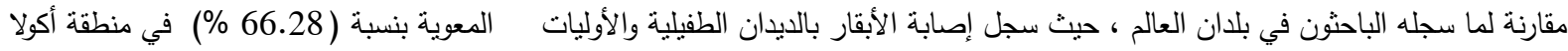

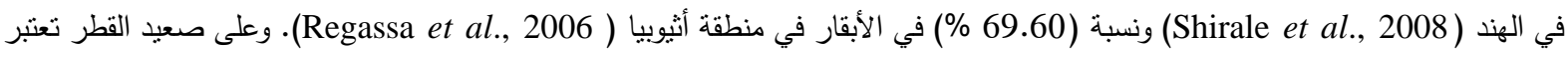

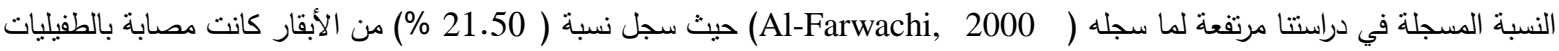

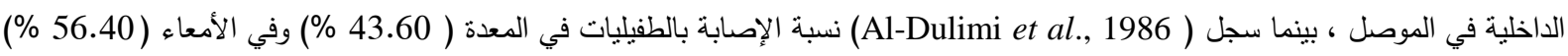

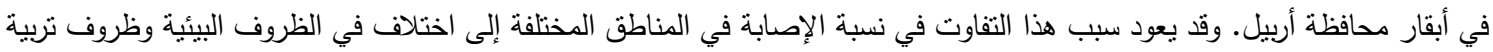
الحيوانات ونظافة الحقل فضلا عن اختلاف في أعداد الحيوانات الدفحوصة وأعمارها.

وأوضحت الننائج أن هناك فرق معنوي في نسب الإصابة للايدان الطفيلية والأوليات المعوية خلا أثنهر الدراسة ، فقد سجلت أعلى نسبة إصابة

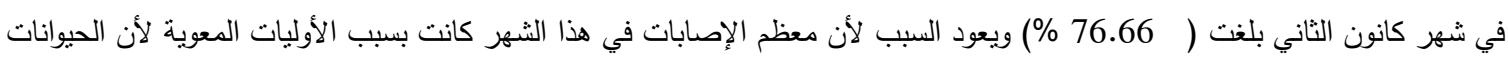

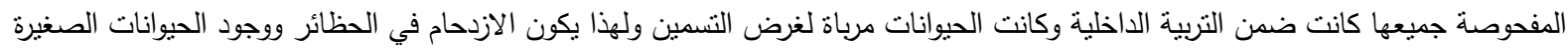

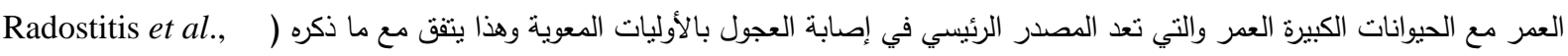

وأثنارت الننائج إلى أن أدنى نسبة إصابة بالديدان الطفيلية كانت في شهر شباط حيث بلغت ( 23.68 \%) وذللك لأنه حصل انخفاض شديد في

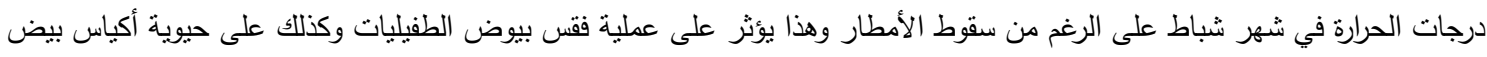

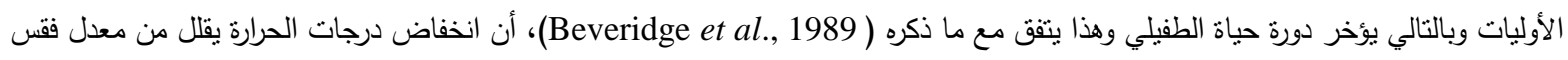

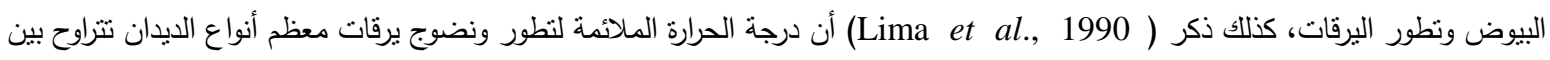
. $32-19$

وتبين من خلال هذه الدراسة بأن الأبقار تصاب بأصناف مختلفة من الديدان الطفيلية مع الأوليات المعوية مع وجود فرق معنوي في نسب الإصابة

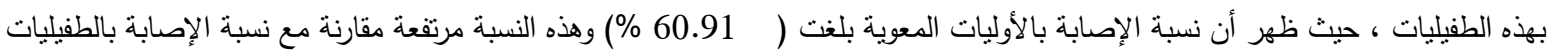

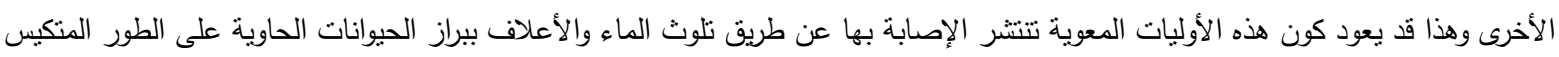

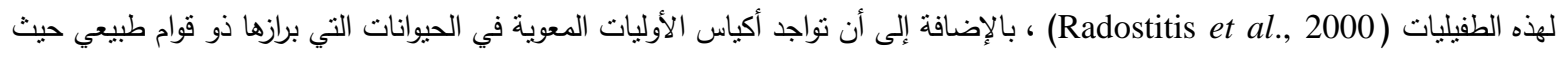

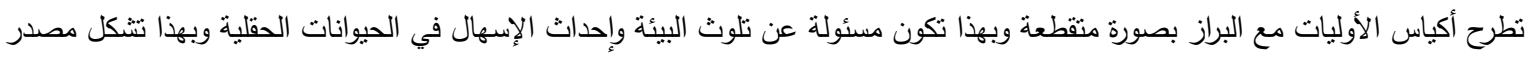

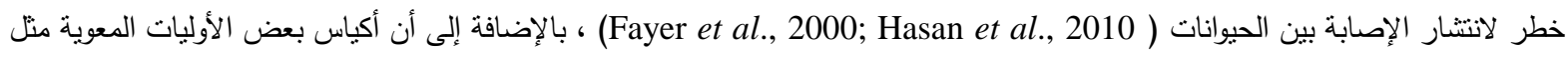

Cryptosporidium spp. داخل جسم الحيوان المصاب (Urquhart et al., 2003).

بينما ظهرت نسبة الإصابة بالديدان الاسطوانية (49.42 \%) وكانت هذه النسبة أعلى مقارنة مع نسبة الإصابة بالديدان الثريطية والمنقوبات التي بلغت (1.72 \%) و (17.24 \%) على التوالي وقد يعود السبب إلى كون دورة حياة الايدان الاسطوانية مباشرة ولا تحتاج إلى مضائف وسطية

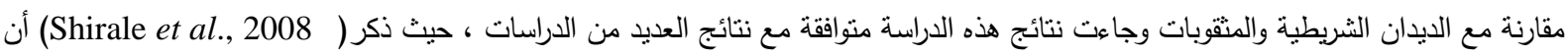

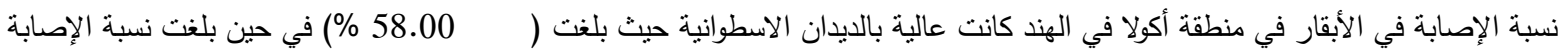

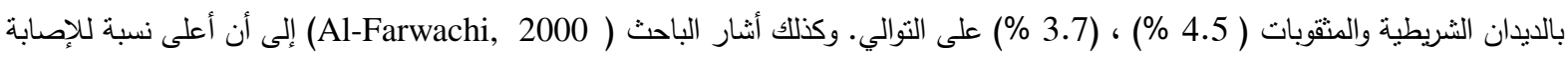
في الأبقار في الموصل كانت بالايدان الاسطوانية (17.5 \%) وأوطأ نسبة للإصابة هي بالديدان الثريطية (0.5 \%

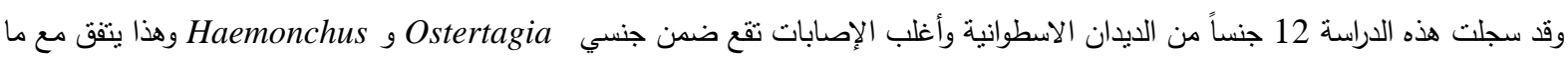

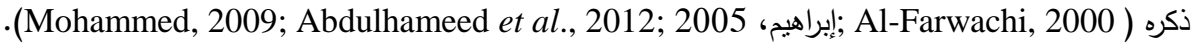

كما وأظهرت النتائج أن الأبقار مصابة بديدان وان Capillaria bovis والتي لم تسجل من قبل في مدينة الموصل وكانت نسبة الإصابة بها

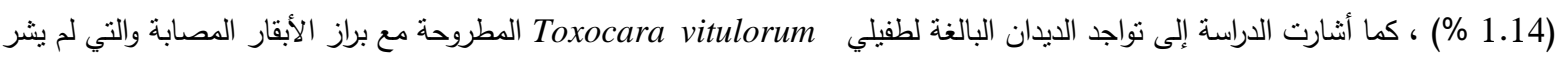


Partovtoma and إليها من قبل في مدينة الموصل ، حيث توفر الظروف المناخية الملائمة لنمو هذا الطفيلي من حيث الرطوبة والحرارة .(Adiwinata, 1993; Aydin et al., 2006

وقد سجلت الدراسة 5 أجناس من ديدان المنقوبات وكانت أعلى نسبة إصابة بطفيلي Dicrocoelium dendriticum حيث بلغت (8.04 \%)

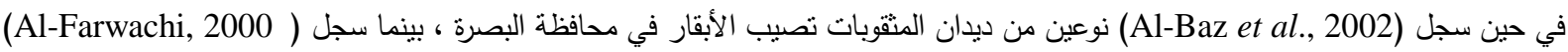
نوع واحد من ديدان المنقوبات التي تصيب الأبقار في مدينة الموصل. في حين لم يسجل الباحث (Al-Dulimi et al., 1986) أب نوع من ديدان المثقوبات أثثاء دراسة مسح لبعض ديدان المعدة والأمعاء في محافظة أربيل وقد يعزى هذا الاختلاف إلى الاختلاف في نظم التربية التي يربى بها الحبوانات بالإضافة إلى الاختلاف في برامج معالجة الحبوانات بمضادات الديدان.

كما سجلت نتائج الدراسة نسبة إصابة الأبقار بالديدان الشريطية من نوع Moniezia expansa (1.72 \% ماندانت النسب،ة مقاربة لما سجله (Al-Baz et al., 2002)

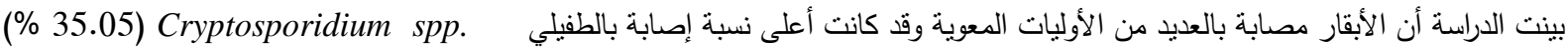

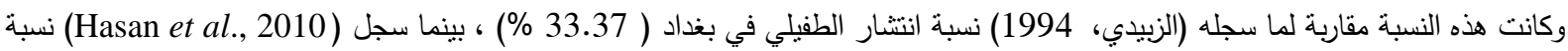
إصابة بهذا الطفيلي عالية في العجول في مدينة الموصل حيث بلغت (52.2 \%). وقد يرجع السبب في تفاوت نسب انتشار الطفيلي في العديد من

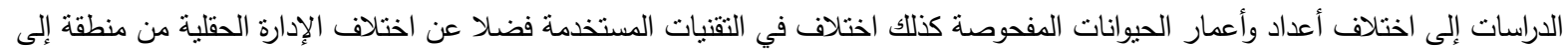
أخرى.والجدير بالذكر أن معظم الإصابات بهذا الطفيلي تحدث عن طريق تلوث مياه الثرب حيث تمثاز أكياس الطفيلي بمقاومته المعظم المعقمات ولها القدرة على البقاء لفترات طويلة في المياه (Schmidt et al., 2000).

وعند تقدير شدة الإصابة بأنواع الديدان الاسطوانية في الأبقار اعتبرت الإصابة خفيفة عندما كان عدد البيوض أقل من 300 بيضة / 1 غم من

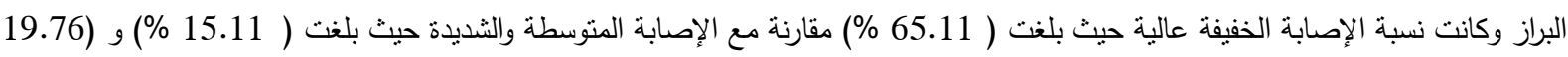

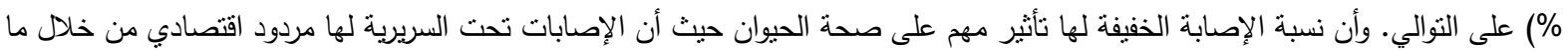

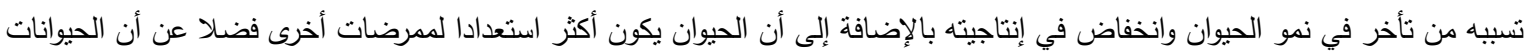
المصابة تكون مصدر لنلوث الحقل وانتشار الإصابة فيه (Criag, 1988).

\section{REFERENCES}

المصادر

Aaiz, N.N. and Mohammed, S.J. (2009): Study for some intestinal protozoa in cows of Al-Qadisiya Province. AlQadisiya J. Vet. Sci., 2(8): 12-19.

Abdulhameed, M.A.; Al-Obaidy, Q.T.; Esmaeal, S.A. and Hussein, KH.J. (2012): Clinical and therapeutic study of gastrointestinal parasites in feedlot calves in Goggeli region. Iraqi J. Vet. Sci., 26(1): 23-27.

Al-Baz, W.J.; Al-Amara, G.Y. and Al-Abood, A.Y. (2002): A survey of some intestinal parasites in bovine of the Basra Province. Bas. J. Vet. Res., 1(1): 37-40.

Al-Dulimi, S.S.; Borhan, A. and Abdul-lattif, M. (1986): Survey of some stomach and intestinal helminthes in cattle of Arbile Province. J. Biological Res., 17(2): 197-203.

Al-Farwachi, M.I. (2000): Occurrence of internal parasites in cattle, Mosul, Iraq. Iraqi J. Vet. Sci., 13(1): 187-191.

Athar, L.A.; Khan, M.N.; Sajid, M.S.; Rehman, T.U. and Khan, I.A. (2011): Cost Benefits Analysis of Anthelmintic Treatment of Cattle and Buffaloes., Pak. Vet. J. 31(2): 149-152.

Aydin, A.; Goz, Y.; Yuksek, N. and Ayaz, E. (2006): Prevalence of Toxocara vitulorum in Hakkari Eastern Region of Turkey. Bull. Vet. Inst. Pulway, 50: 51-54.

Baron, E.J.; Schennane, C. and Tanhenbaum, M. (1984): Comparison of three methods for detection of Cryptosporidium Oocysts in a low prevalence population. J. Clin. Microbiol., 27(1): 223-224.

Baron, E.J.; Peterson, L. and Finegold, S.M. (1994): Diagnostic microbiology. $9^{\text {th }}$ ed., Mosby-Yearbook. Ine. St. Louis, pp:792.

Beveridge, I.; Pullman, A.L.; Martin, R.R. and Barelds, A. (1989): Effects of temperature and relative humidity on development and survival of the free-living stages of Trichostrongylus colubriformis, T. rugatus and T. vitrinus. Vet Parasitol, 33(2):143-153.

Coles, E.H. (1986): Veterinary Clinical Pathology. $4^{\text {th }}$ Ed., W.B., Saunders Company, Philadelphia, pp:377.

Criag, T.M. (1988): Impact of internal parasites on beef cattle. J. Anim. Sci., 66: 1565-1569. 
Fayer, R.; Trout, J.M.; Graczyk, T.K. and Lewis, E.J. (2000): Prevalence of Cryptosporidium, Giardia and Eimeria infections in Post-weaned and adult cattle on three Maryland Farms, Vet. Parasitol, 93(2):12-103.

Foreyt, W.G. (2001): Veterinary parasitology. reference manual. 5th ed., Blackwell Co, pp: 7.

Hasan, M.H.; Butty, E.T. and Al-Taee, A.F. (2010): A study on intestinal protozoa in calves in Nineveh governorate, Iraqi J. Vet. Sci., 24(2): 163-167.

Iqbal, Z.; Akhtar, M.; Khan, M.N. and Riaz, M. (1993): Prevalence and economic significance of haemonchosis in sheep and goats slaughtered at Faisalabad abattoir. Pakistan J. Agric. Sci., 30: 51-53.

Kanyari, P.W.N.; Kagira, J.M. and Mhoma, R.J. (2010): Prevalence of endoparasites in cattle with zoonotic potential within urban and peri-urban areas of Lake Victoria Basin, Kenya. Afri. J. Anim. Biomed. Sci., 4(2): 26-33.

Kassai, T. (1999): Veterinary Helminthology.1st ed., Butterworth Heinemann., pp:190.

Kawan, M.H. (2004): Epidemiological study of infection with intestinal protozoa: Cryptosporidium and Giardia and Eimeria in calves in Baghdad. Iraqi J. Vet. Med., 28(1): 122-133.

Lebbie, S.H.B.; Rey, B. and Irungn, EK. (1994): Small ruminant research and Development in Africa. Proceedings of the second Biennial Conference of the African small Ruminant Research Network. ILCA.: $1-5$.

Leiper, J.W. (1957): Animal parasites and their control. Report to the Government of Iraq. Rome. Fao., P:610.

Lima, J.D.; Lima, W.S.; Guimarães, A.M. and Mallaco, A.M. (1990): Epidemiology of bovine nematode parasites in southeastern Brazil. In: Guerrero J, Leaning WHD (Eds) Epidemiology of bovine nematode parasites in Americas. Proc MSD AGVET symposium, XVI world Buiatrics congress, Salvador, Bahia, Brazil, pp: 49-63.

Mohammed, B.A. (2009): Efficacy of abamectin against gastrointestinal nematodes in calves. Iraqi J. Vet. Sci., 23(1): 167-173.

Partovtoma, S. and Adiwinata, S.G. (1993): The infection of Toxocara vitulorum in cattle and buffalo calves in Selabinta Sukarasa and Suade in the district Sukabumi., Penyakit Hewan, 23: 53-56.

Perry, B.D. and Randolph, T.F. (1999): Improving the assessment of the economic impact of parasitic diseases and their control in production animals. Vet. Parasitol., 84: 145-168.

Polley, L. and Bickis, M.G. (1987): Gastrointestinal Nematode Parasites in Saskatchewan cattle: egg count distributions in beef animals. Can. J. Vet. Res., 51: 465-469.

Radostitis, O.M.; Gay, C.C.; Blood, D.C. and Hinchlif, K.W. (2000): Veterinary Medicine, A text book of disease of cattle, sheep, pigs, goats and horses. $9^{\text {th }}$ ed., W.B., Saunders Company Ltd, London, pp: 566-577.

Regassa, F.; Sori, T.; Dhuguma, R. and kiros, Y. (2006): Epidemiology of gastrointestinal parasites of ruminants in western Oromia . Ethiopia. Intern. J. Appl. Res. Vet. Med., 4 (1): 51 - 57.

Safavi, E.A.; Mohammadi, G.R.; Naghibi, A. and Rad (2011): Prevalence of Cryptosporidium spp. infection in some dairy herds of Mashhad (Iran) and its association with diarrhea in newborn calves. Comp. Clin. Pathol., 20: 103-107.

Schmidt, G.D.; Roberts, L.S. and Janovy, Jr.J. (2000): Foundation Parasitology. $6^{\text {th }}$ ed., London, Madrid, Mexico City, pp: 135-136.

Shah-Fischer, M. and Say, R. (1989): Manual of Tropical Veterinary Parasitology. CAB International; The Technical Center for Agricultural and Rural Cooperation (CTA).

Shirale, S.Y.; Meshram, M.D. and Khillare, K.P. (2008): Prevalence of Gastrointestinal Parasites in Cattle of Western Vidarbha Region. Veterinary World, 1(2): pp: 45.

Soulsby, E.J.L. (1986): Helminths, arthropods and protozoa of domesticated animals., 7th ed., Philadelphia, Bailliere, Tindall, London, pp: 155-156,773.

Urquhart, G.M.; Armour, J.; Duncan, J.L.; Dunn, A.M. and Jennings, F.W. (2003): Veterinary Parasitology., $2^{\text {nd }}$ ed., Black Well Publishing Company, Oxford, pp: 72-73, 233, 276.

Vercruysse, J. and Claerebout, E. (2001): Treatment vs. non -treatment of helminth infections in cattle, defining the thresholds. Vet. Parasitol., 98: 195-214.

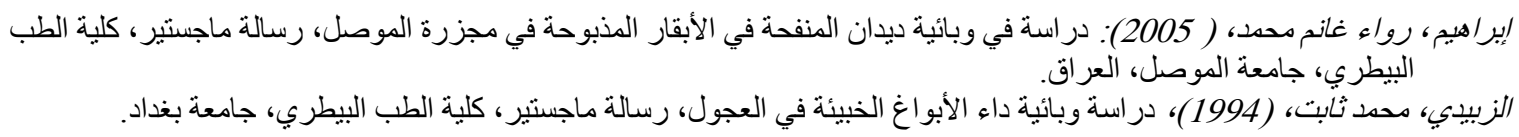

